\title{
LA CONTRIBUCIÓN DE REYNALDO ALARCÓN AL DESARROLLO Y FORTALECIMIENTO DE LA PSICOMETRÍA EN EL PERÚ
}

\author{
RAMÓN LEÓN ${ }^{1}$ \\ Universidad Ricardo Palma, Lima, Perú \\ (RECIBIDO 24/10/2009, ACEPTADO 2/12/2009)
}

\begin{abstract}
RESUMEN
En la presente comunicación se revisa a las contribuciones de Reynaldo Alarcón al desarrollo de la tradición psicométrica en el Perú. Formado bajo la dirección de Walter Blumenfeld, el psicólogo alemán que emigrara al Perú, Alarcón ha desarrollado a lo largo de toda su carrera una activa labor de investigación, en la cual ha estandarizado pruebas psicológicas para nuestro medio y ha explorado aspectos de la mentalidad de los peruanos por medio de inventarios y cuestionarios. Su manual de técnicas de investigación es un libro de excelente factura. Además, se comenta su actual interés por la psicología positiva y, dentro de ella, sus estudios sobre la felicidad.
\end{abstract}

Palabras clave: Reynaldo Alarcón, tests, inventarios, Perú.

\section{ABSTRACT \\ REYNALDO ALARCON CONTRIBUTION TO DEVELOPMENT AND STRENGTHENING OF PSYCHOMETRICS IN PERU}

In this communication the author reviews the contributions of Reynaldo Alarcón to the psychometric tradition in the psychology of Peru. A former student of Walter Blumenfeld, the German psychologist emigrated to Peru in the 1930's, Alarcón is author of a number of investigations in which he standardized questionnaires and inventories and intelligence tests, and explored aspects of the mentality of Peruvians. His text on research techniques and designs is a book of excellent quality. In the frame of this communication the author comments Alarcón's interest for positive psychology, and his recent papers on psychology of happiness.

Keywords: Reynaldo Alarcón, Tests, Inventories, Peru.

\section{INTRODUCCIÓN}

Es larga la tradición psicométrica en el Perú, como lo es en realidad en el mundo entero. Ya lo anotaba, en realidad, hace más de 50 años, Lee J. Cronbach (1957) en su clásico artículo "The two disciplines of scientific psychology". 
Los tests y sus variantes han sido la primera carta de presentación de los psicólogos como profesionales, la primera demostración de lo útil que podía ser la psicología en la solución de algunos de los más importantes problemas sociales. Basta con recordar el significado y la difusión de la escala de evaluación de la inteligencia que crearan Alfred Binet y Theodor Simon en los albores del siglo XX (Carson 2007, Kevles, 2004), así como la importancia concedida por la opinión pública norteamericana a los resultados de evaluaciones psicométricas dados a conocer en el libro The authoritarian personality (Adorno et al. 1950), en los años posteriores a la Segunda Guerra Mundial (Cohen-Cole, 2009).

En el Perú, en ausencia de laboratorios bien dotados y, por lo general, de experimentadores entrenados, la tradición psicométrica se confunde con la psicología misma, como lo demuestra el hecho de que los estudiantes de psicología, tanto ayer como hoy, reciban una gran cantidad de cursos en su formación que tienen que ver con la teoría psicométrica y con los tests mentales, su construcción, su empleo y su interpretación.

Esa tradición ha definido la psicología en nuestro país y tiene entre sus más distinguido representantes a Reynaldo Alarcón.

\section{ORÍGENES DE LA TRADICIÓN PSICOMÉTRICA EN EL PERÚ}

Sin embargo, la tradición psicométrica en el Perú se inicia mucho antes del trabajo de Reynaldo Alarcón. Ya a comienzos del siglo XX podemos encontrar a Hermilio Valdizán (1884-1929), el médico huanuqueño, retornado de Italia después de un fructuoso entrenamiento al lado de la más importante figura de la psiquiatría itálica de esa época, Sancte De Sanctis, desplegando en el que es hoy el Hospital Víctor Larco Herrera una activa labor de aplicación de pruebas psicológicas que incluía los tests de atención de Toulouse y hasta la escala de Binet y Simon. El mismo aportó un test que evaluaba la atención.

Lamentablemente, Valdizán tuvo una muerte prematura que lo sorprendió en medio de una actividad incansable, expresada ya en numerosos estudios y volúmenes y que habría dado lugar a muchos más todavía. Es con razón que Javier Mariátegui considera a Valdizán el gestor del proyecto de la psiquiatría peruana (Mariátegui, 1981).

El discípulo más aprovechado de Valdizán, Honorio Delgado (1892-1969), su sucesor en la enseñanza de la psiquiatría en la Universidad de San Marcos, no mostraba el interés de su maestro por las pruebas psicológicas. Es cierto que les reconocía gran valor, pero por su orientación médica y fenomenológica fueron otras las herramientas utilizadas por él para acceder al estudio de la subjetividad y de la conducta humana.

Sería Walter Blumenfeld (1882-1967), a quien Alarcón ha dedicado un interesante estudio (Alarcón, 1994) y del que fuera discípulo, quien establecería las bases de la tradición psicométrica entre nosotros. Nacido en Alemania, Blumenfeld había desarrollado una carrera científica y académica que lo llevó a ser Ausserordentlicher Professor en la Universidad Técnica de Dresde, en donde tuvo la oportunidad de trabajar con Karl Bühler (1879-1963), una de las grandes figuras de la psicología alemana y mundial de su época. 
Hombre de gran versatilidad conceptual, Blumenfeld se había graduado de Doctor Philosophiae con un original y hasta hoy muy citado trabajo sobre lo que se conoce con el nombre de las "avenidas de Blumenfeld" (Blumenfeld, 1913). Pero, además, a lo largo de su carrera realizó otros trabajos experimentales, investigaciones psicométricas y estudios teóricos, dados a conocer en revistas alemanas y en reuniones científicas (por ejemplo, Blumenfeld, 1934).

De no haber sido por la irrupción del nazismo en Alemania en la primera mitad de los años 30, Blumenfeld jamás habría emigrado al Perú. Su traslado a este país guarda relación con su condición de judío en una nación en la cual el antisemitismo alcanzó niveles en verdad inimaginables hasta ese momento.

En medio de la situación desesperada de haber perdido su condición de docente en la Universidad Técnica de Dresde, Walter Blumenfeld aceptó la invitación que le formulara la Universidad Nacional Mayor de San Marcos para trasladarse al Perú.

Establecido en estas tierras, Blumenfeld encontró en la casa sanmarquina un lugar en el cual proseguir la carrera científica que tan abruptamente había sido interrumpida en Alemania. Pero, no es necesario decir que las condiciones que el científico alemán encontró acá eran distintas, radicalmente diferentes, de aquellas a las que él estaba acostumbrado en su nación de origen.

Podemos imaginarnos los múltiples problemas que enfrentó en nuestro país. Problemas lingüísticos, pues Blumenfeld, un poliglota, no conocía, sin embargo, el castellano; problemas políticos, dado que el antisemitismo también tenía a algunos distinguidos y muy activos representantes en el medio académico sanmarquino; problemas de mentalidad, tan evidentes que es innecesario incidir en ellos; y, finalmente, lo que hoy día llamaríamos problemas de equipamiento.

¿Qué hay que entender por problemas de equipamiento? Pues eso, problemas de equipos. Los laboratorios, aquellos en los que Blumenfeld estaba acostumbrado a trabajar durante la etapa alemana de su vida, disponen de aparatos de medición, de equipos de alta tecnología y de simuladores, que permiten cumplir con tareas docentes y llevar a cabo trabajos de investigación.

Eso es precisamente lo que no había (y, en realidad, lamentablemente sigue sin haber) en nuestro medio. Y fue esa ausencia la que marcó un viraje en la actividad de Blumenfeld quien, impedido de llevar a cabo tareas de naturaleza experimental, decidió incursionar con más frecuencia que hasta entonces en el trabajo de índole psicométrica.

En la realidad peruana que Blumenfeld encontró, él percibió de inmediato la acuciante necesidad de desarrollar instrumentos confiables de medición y de evaluación de las capacidades de estudiantes y de universitarios. En realidad, para eso fue contratado por el gobierno peruano.

Y es así como Blumenfeld, que es presentado (con toda razón) por Alarcón como el introductor de la corriente objetiva en la psicología peruana (Alarcón 2000b), es también si no el iniciador, sí, el que impulsa de manera decidida en nuestro medio la variante psicométrica del trabajo científico. 
Muchos son los discípulos que dejó el maestro alemán entre nosotros. Discípulos en el ámbito de la educación y en el de la psicología. Uno de sus primeros colaboradores fue Miguel Sardón, una figura muy poco mencionada al historiar la psicología entre nosotros.

Pero podemos mencionar también a Violeta Tapia, estudiosa peruana de muy distinguida trayectoria y activa hasta el día de hoy. Otro fue Modesto Rodríguez, durante muchos años profesor de psicología experimental, que desarrolló este curso en varias universidades con las limitaciones que un medio como el nuestro impone a quienes quieren llevar a cabo trabajo experimental.

Otro más, el protagonista de esta comunicación, es Reynaldo Alarcón.

\section{EL TRABAJO DE REYNALDO ALARCÓN}

Formado como psicólogo e investigador bajo la dirección de Walter Blumenfeld, Reynaldo Alarcón ha desplegado desde el inicio de su carrera hasta el día de hoy una actividad ininterrumpida en su frecuencia, comprometida con el país y sus problemas, perseverante en el enfoque psicométrico y atenta a los cambios producidos en el escenario de la psicología mundial.

Desde el inicio de su carrera, en efecto, Alarcón se ha dedicado a la investigación, siempre proclamada como necesaria y siempre presentada como el supremo objetivo de la universidad, pero siempre negligida y postergada por una sociedad como la nuestra que, al no haber experimentado ninguna de las grandes revoluciones que han dado lugar a la modernidad, no valora la ciencia ni concede incentivos al trabajo científico.

La cantidad de trabajos escritos y publicados por Alarcón supera la centena de ítems sin que esto signifique, por cierto, que su carrera científica haya concluido. Por el contrario, su paso a la condición de Profesor Emérito ha ido acompañado de un redoblado trabajo investigatorio expresado en numerosos reportes de investigación tanto en el país como en el extranjero, y en la publicación de un muy leído y reeditado manual de técnicas de investigación, que lleva por título Métodos y diseños de investigación del comportamiento (Alarcón 1991).

Como autor, Alarcón ha hecho uso de muy variadas formas de la comunicación científica: desde la preparación de las perecibles separatas de clase pasando por la edición de memorias de congresos (Alarcón et al., 1975), el folleto, el ensayo, el artículo periodístico y el dirigido a la revista especializada (e.g. Alarcón, 2004a, 2004b), la recensión y el reporte de investigación (e.g. Alarcón, 2000a, 2006a, 2006b) hasta el manual (Alarcón 1991) y finalmente el tratado (Alarcón, 2000b).

El manual sobre investigación del comportamiento merece una mención especial. Se trata de un unicum en la literatura psicológica peruana. Por lo general, los psicólogos que se dedican a la labor investigativa suelen recurrir a los manuales internacionales sobre el tema, de los cuales hay muchos en el mercado. Algunos, como el de Selltiz et al. (1974) y el de Kerlinger (1975) han sido considerados casi biblias, a las que recurrían todos, neófitos e iniciados. 
Lo cierto, sin embargo, es que el libro de Alarcón compite con ellos con el "valor agregado" de ser un texto escrito por uno de nosotros, que tiene una amplia experiencia investigatoria, que escribe en nuestro lenguaje (de modo tal que no necesitamos de traductores y de interpretaciones intuitivas acerca de lo que autores de otras latitudes y realidades idiomáticas quieren decir), y que se distingue por su nivel de actualización.

Pero, dejando de lado a este manual, debe mencionarse a un reactivo psicométrico que en el Perú está estrechamente vinculado al nombre y al trabajo de Alarcón: la Prueba de Madurez Mental de California, un reactivo de aplicación tanto individual como colectiva, que Alarcón adaptó y normalizó para nuestro medio (Alarcón, 1961a, 1961b).

Hoy más bien olvidada, la Prueba de Madurez Mental de California era un reactivo muy conocido y empleado por los psicólogos peruanos hasta inicios de los años 1980. Cuando alguien que preparaba su tesis deseaba "controlar" la variable inteligencia de su muestra, por lo general recurría a esta escala, una de las pocas (si no la única) con normas para nuestro medio.

Podría hacerse una historia del empleo de esta escala entre nosotros, que permitiría verificar la frecuencia de su uso, la importancia que se le concedió en su momento, y por tanto el valioso aporte que Reynaldo Alarcón rindió a la comunidad psicológica nacional al poner a su disposición un instrumento de tanto valor.

Ciertamente, no ha sido la Prueba de Madurez Mental de California el único reactivo que Alarcón estandarizara para nuestro medio. Ya a fines de 1959 e inicios de los años 1960 dio a conocer en el Boletín del Instituto Psicopedagógico Nacional la revisión del Test Colectivo de Inteligencia PV de Th. Simon (Alarcón, 1951), y años después trabajaría activamente con el Inventario de Personalidad de Hugh M. Bell (Alarcón, 1962).

La investigación psicométrica de Alarcón no se ha perdido en los recovecos de la psicología. ¿No ha leído alguien algún trabajo publicado en una importante revista internacional acerca de si es mejor para el estado de ánimo hacer jogging con su mascota o hacerlo sin ella?, ¿no nos hemos topado, con sorprendente frecuencia, con trabajos que tratan de temas intrascendentes no solo para nuestra sociedad, sino para aquella en la cual vive y trabaja el investigador?

Se puede afirmar en defensa de estos trabajos de investigación que la ciencia necesita de todo: de lo trascendente y de lo banal, de lo profundo y de lo trivial, de lo intemporal así como de aquello que se circunscribe al aquí y al ahora. Eso es cierto, y si se ha presentado los ejemplos previos no ha sido con un ánimo condenatorio sino con propósitos de comparación, pues Reynaldo Alarcón ha investigado temas que sí pueden ser considerados trascendentes en una realidad como la nuestra, tan compleja, tan desafiante, tan cambiante y tan difícil, como ocurrió en uno de los congresos (el único realizado en esa ciudad) de la Sociedad Interamericana de Psicología, en Bogotá, en 1974.

Uno de los simposios incluidos en el programa al cual asistió toda la delegación peruana presente en ese evento fue el dedicado a exponer un conjunto de investigaciones sobre la adolescencia en el Perú promovidas por Reynaldo Alarcón entre psicólogos que, con sus trabajos, se titulaban de tales en esta casa de estudios. Los investigadores exponían los resultados de sus investigaciones psicométricas en adolescentes peruanos, trabajando con 
la Lista de Problemas de Mooney, el inventario de preferencias personales de Edwards y otros reactivos más.

Referimos esto a modo de ejemplo a fin de acreditar lo que hemos señalado. Es decir, no solo a la ininterrumpida actividad investigatoria de Alarcón, sino también a la orientación de los estudios que él llevaba a cabo o que él estimulaba. La realidad peruana ha estado presente en ellos desde siempre.

Pero no hay una realidad peruana, sino muchas. Para concentrarnos en Lima, una es la realidad de un distrito como San Isidro o como La Molina y otra la de un distrito como San Juan de Lurigancho o Independencia. Hay entre ellos diferencias abismales, casi inconcebibles si se piensa que todos forman parte de eso que se llama Lima Metropolitana.

Mientras que algunos sociólogos e historiadores se han inclinado a estudiar la clase alta (e.g. Rizo-Patrón-Boylan \& Aljovín de Losada, 1998; Rizo-Patrón-Boylan, 2000; Kogan, 2009) y otros la clase media (e.g. Portocarrero 1998; Gamero \& Zeballos, 2003; Plaza 2007), Alarcón se concentró en el estudio de la pobreza, uno de los fenómenos más distintivos de la realidad peruana (Alarcón, 1986).

Su libro sobre la pobreza y el subdesarrollo sigue siendo hoy, a 23 años de su aparición, el más importante y coherente esfuerzo desarrollado por los psicólogos peruanos para entender el fenómeno de la pobreza en nuestra sociedad.

Si bien se trata de un estudio de naturaleza teórica, quien lo lea puede reconocer de inmediato que Alarcón sustenta la gran mayoría de sus afirmaciones en datos fácticos derivados de sus estudios de naturaleza psicométrica.

En los últimos años, inspirado en las teorías de Rogelio Díaz-Guerrero, el conocido psicólogo mexicano con quien lo uniera una amistad de décadas, Alarcón ha comenzado a investigar la mentalidad de los peruanos a través de las premisas histórico-socioculturales de las que hablara Díaz-Guerrero, habiendo dado a conocer hasta ahora solo alguna de la abundante información que sé que ha sido el fruto de sus investigaciones. Tres trabajos pueden ser consultados sobre el particular: "Premisas histórico-socioculturales de la juventud peruana: obediencia filial y virginidad", publicado en la Revista Latinoamericana de Psicología (Alarcón 2005a); "Premisas histórico-socioculturales de la juventud peruana (Lima): machismo, rigidez cultural y abnegación" y "Premisas histórico-socioculturales de la juventud peruana (Lima): statu quo familiar, relaciones familiares, honor familiar y respeto sobre amor", estos dos últimos dados a la luz en Teoría e investigación en Psicología (Alarcón 2004c, 2006b).

\section{LA PSICOLOGÍA TRANSCULTURAL}

Pero, además, Alarcón se ha mantenido al par que perseverante en el enfoque psicométrico atento a los cambios que se producen en la psicología internacional. Ese es precisamente el caso de lo ocurrido en los años 70 cuando una importante tendencia de la psicología contemporánea apuntaba con entusiasmo a lo que se conoce como psicología transcultural. 
En efecto: fueron varias conferencias de Alarcón las dictadas sobre el tema, y quien busque en su bibliografía encontrará referencias precisas sobre el particular (Anónimo, 2003). En realidad, Alarcón no solo se interesó por la psicología transcultural al aliento de lo que ocurría en el mundo de la psicología en aquella época, sino también porque fue influido por su maestro Blumenfeld, que ya a fines de los años cuarenta y comienzos de los cincuenta hablaba nada menos que de "vergleichende Ethnopsychologie", algo que puede ser traducido como "etnopsicología comparada" (Blumenfeld, 1952).

Las ideas de Blumenfeld, en su momento, y las de Alarcón, presentadas en conferencias y en publicaciones, cobran gran actualidad en nuestros días, cuando se habla de la psicología cultural (Cole, 1999; Valsiner, 2007).

¿Qué duda cabe que el Perú es un país ideal para la investigación transcultural? Hoy lo sabemos mucho mejor que antes. Por décadas, casi a lo largo de todo un siglo podríamos decir, hemos venido insistiendo en los aspectos que hacían del Perú una sociedad homogénea, tratando de encontrarlos en el idioma castellano y en el predominio de la religión católica.

Estamos ahora de retorno de esa idea, y, por el contrario, reconocemos que pocos países son tan diversos como el nuestro (Degregori, 2000). Se comienza además a reconocer en esa diversidad un elemento constitutivo de la identidad nacional, y también la inmensa riqueza que ella supone. Pero la diversidad también plantea grandes problemas, como los de exclusión social y los de la educación intercultural, acerca de los cuales la sociedad peruana todavía no encuentra una solución medianamente operativa.

De otro lado, los cambios producidos en el Perú en los últimos treinta o cuarenta años han sido de tal magnitud que el investigador, sea éste psicólogo, sociólogo o antropólogo, tiene que replantear sus esquemas interpretativos y sus prioridades en materia de estudio.

Así, el Perú ha dejado de ser un país rural para ser una sociedad cada vez más urbana. Los problemas urbanos, los problemas de las grandes ciudades han comenzado a cobrar una importancia que antes no poseían. Regiones enteras como la selva, en el pasado apenas exploradas y escasamente consideradas como temas de interés, hoy poseen un significado que nadie puede discutir.

Si, como lo señala Rénique (2008), los grandes problemas que concentraban la atención de los estudiosos a fines del siglo XX giraban en torno al "desborde popular y la crisis del Estado", "el mito del progreso" en las poblaciones andinas, la "modernidad popular" y la "choledad", y la "otra modernidad" y la "plebe urbana", hoy la agenda se ha enriquecido con los problemas derivados de una regionalización que avanza a trompicones, el surgimiento de elites locales que reclaman un mayor poder de decisión, la fuerza cada vez mayor del narcotráfico, la conciencia atemorizante entre la ciudadanía de la ubicuidad de la violencia, la corrupción y las nacientes reivindicaciones étnicas.

No es fácil, por tanto, estar a la altura de los desafíos que una sociedad como la peruana plantea a aquel que quiere investigarla. Alarcón ha hecho lo suyo abordando los temas de la pobreza, explorando las cambiantes opiniones en materia de religión entre los jóvenes así como sus motivaciones (Alarcón, 1969, 1978), y más recientemente lo referente a aspectos de nuestra mentalidad. Con esto, como se podrá deducir, Alarcón 
ha hecho incursiones en un tema de complejidad y profundidad oceánicas: el tema de la identidad nacional.

Poco es aún lo que la psicología ha contribuido al estudio de la identidad nacional. Los trabajos de Alarcón, los ya publicados y aquellos que tiene en curso de preparación, constituyen una valiosa fuente de datos y hallazgos que permitirán -más temprano que tarde- formular algunas apreciaciones con sustento acerca de lo que somos y de lo que sentimos los peruanos por el hecho de serlo.

\section{REYNALDO ALARCÓN Y LA PSICOLOGÍA POSITIVA EN EL PERÚ}

Y el segundo ejemplo de la permanente actualización de Alarcón en lo que se refiere a la temática de sus trabajos, lo tenemos en su actual interés por la psicología positiva, cuya originalidad, como él mismo señala, "reside en que focaliza su estudio en áreas inexploradas del psiquismo humano, conectadas con el bienestar psicológico, la realización del individuo como persona y el desarrollo de virtudes cívicas para vivir en armonía en una sociedad plural, que son temas de nuestro tiempo" (Alarcón, 2008: 8).

Se trata de una corriente cada vez con mayor fuerza al interior de la psicología, que trata de estudiar y destacar la importancia de emociones de matiz positivo, tales como la felicidad, el bienestar, la alegría. Nombres como los de Seligman (2004) y Csikszentmihalyi (1998), entre otros, están vinculados a este enfoque de la vida psicológica.

Se podrá decir que se trata de un tema de estudio de interés sobre todo para los psicólogos clínicos, los psicoterapeutas y los psicólogos educacionales, por último. Pero, en realidad, la psicología positiva termina siendo también un enfoque relevante para áreas como la producción industrial, la optimización del trabajo empresarial, la creatividad artística y científica.

En los últimos años Alarcón ha venido investigando esta área concentrándose en el estudio de la felicidad, tema complejo, elusivo y controversial (e.g. Alarcón, 2000a, 2002, 2005b). Sus trabajos recién comienzan a aparecer y un libro, uno más en su carrera, está muy, muy próximo a aparecer en el sello editorial de la Universidad Ricardo Palma.

Además de eso, ha estimulado de modo decidido la constitución de la Sociedad Peruana de Psicología Positiva, la misma que ha llevado a cabo con éxito el Primer Coloquio Peruano de Psicología Positiva en las postrimerías del 2008.

\section{LA PARTICIPACIÓN INSTITUCIONAL DE REYNALDO ALARCÓN}

Esto nos lleva a tratar de un aspecto algo lejano al tema de esta comunicación, pero que es relevante para entender la obra de Alarcón. Se trata de la participación suya en instituciones de gran significado para la vida de nuestro país.

Reynaldo Alarcón se ha desempeñado en calidad de funcionario ejecutivo en el Consejo Nacional de Ciencia y Tecnología (CONCYTEC), estimulando el trabajo de investigación, especialmente en el ámbito de las ciencias sociales. Se desempeñó, asimismo, como Director del lamentablemente desaparecido Instituto Nacional de Investigación y Desarrollo de 
la Educación (INIDE), el segundo ensayo más importante en la historia de nuestro país de llevar a cabo sustantivas reformas educativas, después del Instituto Psicopedagógico Nacional, creado a fines de la década de los 1940, y en el cual su maestro, Walter Blumenfeld, ocupara un lugar de gran importancia.

Y, por último, debe destacarse su presencia en el Comité Asesor Nacional de la más importante institución de salud mental en el Perú, el Instituto Nacional de Salud Mental Honorio Delgado-Hideyo Noguchi. Proyecto acariciado por varias generaciones de psiquiatras peruanos, el Instituto fue creado en 1982 con el propósito de estudiar la realidad psicológica y conductual de los peruanos. Su fundador y primer director, el doctor Javier Mariátegui (1929-2008), logró comprometer a un conjunto de personalidades para que sirvieran como orientadores de la novísima institución. Una de ellas, al lado de psiquiatras, historiadores, filósofos y sociólogos, fue Reynaldo Alarcón.

\section{PALABRAS FINALES}

Estamos pues ante una figura de la psicología peruana, que a lo largo de casi 60 años de trabajo ininterrumpido ha venido ofreciendo de manera sistemática, estudios rigurosos y creativos al mismo tiempo sobre temas de relevancia para la sociedad peruana, así como para la teoría psicológica internacional. Y esto lo ha hecho con un rasgo que es la impronta de todo su trabajo: de manera silenciosa, sin aspavientos ni luces de marquesina, algo que suele ejercer una atracción morbosa entre muchos de nosotros.

Con sus numerosos trabajos, sus reiteradas conferencias, su labor docente (iniciada en la Universidad de San Marcos y culminada con el título de Profesor Emérito, pero proseguida en otras casas de estudio hasta hoy, a pedido expreso de las autoridades de ellas) en pregrado y posgrado, con sus libros, con sus investigaciones en marcha, pero también con sus conversaciones informales y estímulos, Reynaldo Alarcón ha influido y sigue influyendo de modo sustantivo en el curso por el cual transita la psicología peruana.

\section{REFERENCIA}

Conferencia en la I Jornada Interuniversitaria de Innovación en Investigación Psicométrica "De la práctica científica a la labor profesional”, organizada por el Gabinete de Psicometría del Instituto de Investigaciones Psicológicas de la Facultad de Psicología de la Universidad Nacional Mayor de San Marcos, el 23 y 24 de octubre de 2009.

\section{REFERENCIAS BIBLIOGRÁFICAS}

1. Adorno, Th. W.; Frenkel-Brunswik, E.; Levinson, D. \& Sanford, N. (1950). The authoritarian personality. New York: Harper.

2. Alarcón, R. (1951). Resultados de la aplicación del Test Colectivo P. V. del Dr. Th. Simon, en 185 alumnos de educación primaria. Revista de Educación Nacional, 5, 267-294. 
3. Alarcón, R. (1961 a). Estandarización de la Prueba de Madurez Mental de California. Serie Intermedia, 1950-S. Boletín del Instituto Psicopedagógico Nacional, 16 (4), 3-35.

4. Alarcón, R. (1961b). Perfil mental de los adolescentes determinado a base de la Prueba de Madurez Mental de California. Educación, 15(24), 85-98.

5. Alarcón, R. (1962). Desajustes de la personalidad investigados mediante el Inventario de Hugh M. Bell. Boletín del Instituto Psicopedagógico Nacional, 17(5), 3-33.

6. Alarcón, R. (1969). Objetivos vitales en adolescentes de distintos niveles socioeconómicos. Revista Latinoamericana de Psicología, 1, 55-61.

7. Alarcón, R.; Infante, J.; Ponce, C. \& Bibolini, A., (comps.) (1975). La investigación psicológica en el Perú. Memorias del Primer Congreso Peruano de Psicología. Lima: Sociedad Peruana de Psicología.

8. Alarcón, R. (1978). Actitudes hacia la religión en un grupo de estudiantes universitarios del Perú. Revista Latinoamericana de Psicología, 10, 193-209.

9. Alarcón, R. (1986). Psicología, pobreza y subdesarrollo. Lima: INIDE.

10. Alarcón, R. (1991). Métodos y diseños de investigación del comportamiento. Lima: Universidad Peruana Cayetano Heredia [2da. edición, ampliada, Lima, Universidad Ricardo Palma, 2008)].

11. Alarcón, R. (1994). El pensamiento psicológico de Walter Blumenfeld. Lima: CONCYTEC.

12. Alarcón, R. (2000a). Variables psicológicas asociadas con la felicidad. Persona, 3, $147-157$.

13. Alarcón, R. (2000b). Historia de la psicología en el Perú. De la Colonia a la República. Lima: Universidad Ricardo Palma.

14. Alarcón, R. (2002). Fuentes de felicidad: ¿qué hace feliz a la gente? Revista de Psicología (Lima, PUCP), 20, 169-196.

15. Alarcón, R. (2004a) Medio siglo de psicología latinoamericana: una visión de conjunto. Interamerican Journal of Psychology, 38(2), 307-316.

16. Alarcón, R. (2004b). 50 años de psicología interamericana: evaluación y perspectivas. Interamerican Journal of Psychology, 38(2), 150-152.

17. Alarcón, R. (2004c). Premisas histórico-socioculturales de la juventud peruana (Lima): machismo, rigidez cultural y abnegación. Teoría e Investigación en Psicología, 13, 7-31.

18. Alarcón, R. (2005a). Premisas histórico-socioculturales de la juventud peruana: obediencia filial y virginidad. Revista Latinoamericana de Psicología, 37, 81-94.

19. Alarcón, R. (2005b). Efectos de los desniveles socioeconómicos sobre la felicidad. Teoría e Investigación en Psicología, 14, 91-112. 
20. Alarcón, R. (2006a). Desarrollo de una escala factorial para medir la felicidad. Interamerican Journal of Psychology, 40, 1, 95-102.

21. Alarcón, R. (2006b). Premisas histórico-culturales de la juventud peruana (Lima): statu quo familiar, relaciones intrafamiliares, honor familiar y respeto sobre amor. Teoría e Investigación en Psicología, 16, 9-32.

22. Alarcón, R. (2008). Psicología positiva: un enfoque emergente. Temática Psicológica, 4 (1), 7-20.

23. Anónimo (2003). Publicaciones del Dr. Reynaldo Alarcón. Teoría e Investigación en Psicología, 11, 177-188.

24. Blumenfeld, W. (1913). Experimentelle Untersuchungen über die scheinbare Grösse im Sehraume. Zeitschrift für Psychologie, 65, 241-404.

25. Blumenfeld, W. (1934). Intelligenzprüfungen und Denkpsychologie. En: Gundlach, H., ed., Applied psychology (vol. 8, The Eighth Congress, Prague 1934), Londres y Nueva York, Routledge, 663-670 [reproducción facsimilar de los Comptes Rendus de la VIIIe. Conférence Internationale de Psychotechnique, celebrada en Praga del 11 al 15 de setiembre de 1934].

26. Blumenfeld, W. (1952). Erfahrungen mit Intelligenz- und charakterologischen Tests in Peru und ihre Beziehungen zum Problem der vergleichenden Ethnopsychologie. En: Baumgarten, F., ed., La psychotechnique dans le monde modern. Compte rendu du IX. Congrès International du Psychotechnique, Berna, 12.-17. Sept. 1949, París, Presses Universitaires de France, 517-527.

27. Carson, J. (2007). The measure of merit. Talents, intelligence, and inequality in the French and American republics, 1750-1940. Princeton y Oxford: Princeton University Press.

28. Cohen-Cole, J. (2009). The creative American: Cold war salons, social science, and the cure for modern society. Isis, 100 (2), 219-262.

29. Cole, M. (1999). Psicología cultural. Una disciplina del pasado y del futuro. Madrid: Morata.

30. Cronbach, L. J. (1957). The two disciplines of scientific psychology. American Psychologist, 12, 671-684.

31. Csikszentmihalyi, M. (1998). Fluir (Flow). Una psicología de la felicidad. Barcelona: Kairos.

32. Degregori, C. I., ed. (2000). No hay país más diverso. Compendio de antropología peruana. Lima: Red para el Desarrollo de las Ciencias Sociales.

33. Gamero, J. \& Zeballos, M., eds. (2003). Perú hoy. La clase media ¿existe? Lima: DESCO.

34. Kerlinger, F. N. (1975). Investigación del comportamiento. Técnicas y metodología. México DF: Interamericana.

35. Kevles, D. J. (2004). In the name of eugenics. Genetics and the uses of human heredity. Londres - Cambridge, Mass.: Harvard University Press. 
36. Kogan, L. (2009). Regias y conservadoras. Mujeres y hombres de clase alta en la Lima de los noventa. Lima: Fondo Editorial del Congreso del Perú.

37. Mariátegui, J. (1981). Hermilio Valdizán. El proyecto de una psiquiatría peruana. Lima: Librería Editorial Minerva.

38. Plaza, O., (Coord.). (2007). Clases sociales en el Perú. Visiones y trayectorias. Lima: Pontificia Universidad Católica del Perú.

39. Portocarrero, G. (1998). Las clases medias: entre la pretensión y la incertidumbre. Lima: Sur - Oxfam.

40. Rénique, J. L. (2008). Esperanza y fracaso en la historia del Perú. En: Pásara, L., ed., Perú en el siglo XXI, Lima, Pontificia Universidad Católica del Perú, 15-45.

41. Rizo-Patrón Boylan, P. (2000). Linaje, dote y poder. La nobleza de Lima de 1700 a 1850. Lima: Pontificia Universidad Católica del Perú.

42. Rizo-Patrón-Boylan, P. \& Aljovín de Losada, C. (1998). La elite nobiliaria de Trujillo de 1700 a 1830. En: O'Phelan Godoy, S. \& Saint-Geours, Y., (Comps)., El norte en la historia regional. Siglos XVIII - XIX, Lima: Instituto Francés de Estudios Andinos - Centro de Investigación y Promoción del Campesinado, 241-293.

43. Seligman, M. E. P. (2004). La auténtica felicidad. Buenos Aires: Vergara.

44. Selltiz, C.; Jahoda, M.; Deutsch, M. \& Cook, S. W. (1974). Métodos de investigación en las relaciones sociales. Madrid: Rialp.

45. Valsiner, J. (2007). Culture in minds and societies: Foundations of cultural psychology. Thousand Oaks CA: Sage. 